\title{
Objective analysis of midterm outcomes of conventional and hybrid aortic arch repair by propensity-score matching
}

\author{
Arudo Hiraoka, MD, Genta Chikazawa, MD, PhD, Toshinori Totsugawa, MD, PhD, Kentaro Tamura, MD, \\ Atsuhisa Ishida, MD, Taichi Sakaguchi, MD, PhD, and Hidenori Yoshitaka, MD, PhD
}

\section{ABSTRACT}

Objective: The aim of this study is to evaluate the objective outcomes of conventional total aortic arch repair (CTAR) and hybrid arch repair by using propensityscore matching to reduce selection bias.

Methods: Between January 2006 and April 2016, 470 consecutive patients underwent isolated aortic arch repair (excluding hemiarch or partial arch reconstruction, and cases with concomitant cardiac surgeries) at a single cardiovascular institute. We categorized 337 total aortic arch repair with antegrade cerebral perfusion under circulatory arrest as the CTAR group and 58 hybrid aortic arch repair (HAR) with thoracic endovascular aortic repair as the HAR group. Seventy-five patients with scheduled and staged thoracic endovascular aortic repair after total aortic arch repair with elephant trunk were excluded. Then, we compared early and midterm outcomes between the propensity-matched group (43 CTAR vs HAR pairs).

Results: There were no significant differences in 30-day and operative deaths between the CTAR and HAR groups $(4.7 \%$ [2/43] vs $7.0 \%$ [3/43]; $P=.4142$ and $11.6 \%$ [5/43] vs $16.3 \%$ [7/43]; $P=.5637)$. Although there were no significant differences in the incidences of other major complications, permanent stroke was observed more frequently in the HAR group $(0 \%$ [0/43] vs $11.6 \%$ [5/43]; $P=.0064)$ compared with the CTAR group. Matching analysis, however, revealed an equivalent 5 -year survival rate between the CTAR and HAR groups $(80.5 \%$ vs $59.9 \% ; P=.1300)$.

Conclusions: Matching analysis revealed a significantly greater incidence of stroke in the HAR group but equivalent midterm outcomes in the hybrid group compared with the CTAR group. (J Thorac Cardiovasc Surg 2017;154:100-6)

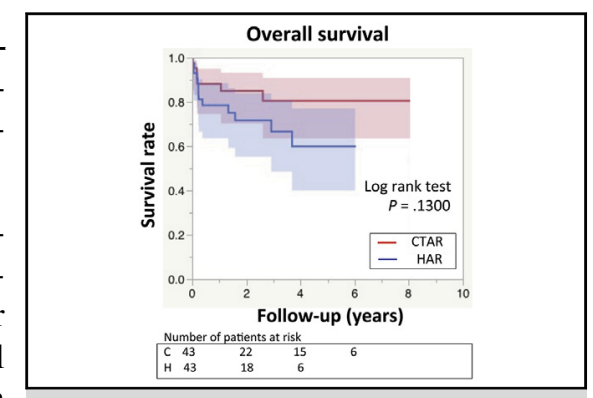

Matched analysis reveals equivalent survival rate between conventional and hybrid groups.

Central Message

Matching analysis revealed equivalent midterm outcomes in the hybrid arch repair groups compared with the conventional arch repair group.

\begin{abstract}
Perspective
Matching analysis revealed a significantly greater incidence of postoperative stroke but equivalent midterm outcomes in the hybrid arch repair group compared with the conventional arch repair group. In a high-risk population, hybrid approaches have the potential to be alternatives to a conventional approach. Further development, however, is required for hybrid repair to become a superior option.
\end{abstract}

See Editorial Commentary page 107.

See Editorial page 98.
Conventional surgical total aortic arch repair (CTAR) with the use of antegrade cerebral perfusion under moderate hypothermia is an established procedure with excellent 5-year survival reported to be $70 \%$ to $90 \% .{ }^{1-6} \mathrm{CTAR}$, however, is not necessarily an optimal strategy, especially for high-risk patients, considering the invasiveness of surgery. Therefore, hybrid aortic arch repair (HAR) with thoracic endovascular

From the Department of Cardiovascular Surgery, The Sakakibara Heart Institute of Okayama, Japan.

Received for publication May 27, 2016; revisions received Nov 18, 2016; accepted for publication Dec 12, 2016; available ahead of print March 14, 2017.

Address for reprints: Arudo Hiraoka, MD, Department of Cardiovascular Surgery, The Sakakibara Heart Institute of Okayama, 2-5-1 Nakaicho, Kita-ku, Okayama 700-0804, Japan (E-mail: bassbord1028@yahoo.co.jp).

0022-5223/\$36.00

Copyright (c) 2017 by The American Association for Thoracic Surgery

http://dx.doi.org/10.1016/j.jtcvs.2016.12.060 aortic repair (TEVAR) has been introduced in the last decade for patients with comorbidities and frailties. ${ }^{7-16}$ The safety of hybrid TEVAR securing enough proximal landing zone and requiring supra-aortic rerouting bypass is still, however, controversial. We previously reported satisfactory midterm outcomes of CTAR (7-year survival $=90.1 \%$ ), compared with HAR with TEVAR, but the preoperative patient background in that report was significantly different because hybrid approaches had

Scanning this QR code will take you to supplemental figures, table and a video for the article.






\section{Abbreviations and Acronyms}

CTAR = conventional surgical total aortic arch repair

HAR = hybrid aortic arch repair

PTFE = polytetrafluoroethylene

TEVAR $=$ thoracic endovascular aortic repair

been used for high-risk patients. ${ }^{17}$ Patient background makes it difficult to evaluate the veritable superiority in outcomes, and there are still limited studies regarding riskadjusted comparison between conventional and hybrid approaches. ${ }^{18,19}$ The aim of this study is to evaluate the objective outcomes of CTAR and HAR by the use of propensity-score matching to reduce selection bias.

\section{PATIENTS AND METHODS}

Between January 2006 and April 2016, 470 consecutive patients underwent isolated aortic arch repair (excluding hemiarch or partial arch reconstruction, and cases with concomitant cardiac surgeries) at the Sakakibara Heart Institute of Okayama in Japan. In the present study, 75 patients with staged and scheduled TEVAR after reconstruction of arch vessels with elephant trunk for extended lesion were excluded, because this procedure is a useful option for extended lesion but controversial as a hybrid option. Of those remaining, CTAR with antegrade cerebral perfusion under moderate hypothermia was performed for 337 patients and debranching with TEVAR (including 1, 2, and total rerouting) in 58 patients, including 4 patients with chimney technique for zone 0 . The patients with an isolated lesion at the descending aorta were not included in the group. Patients who underwent debranching with TEVAR and chimney technique were categorized as HAR group to aortic arch lesion.

In our institute, conventional open repair basically was considered as the first choice. We also selected the respective hybrid approaches for high-risk patients, based on the patient frailty, anatomically broad lesion, and aortic shagginess. This study is a retrospective risk-adjusted comparison of early and midterm clinical outcomes between CTAR and respective hybrid approaches with propensity-score matching. The project was approved by the institutional review board of the Sakakibara Heart Institute of Okayama, on April 1, 2016. Since it has been reported previously, the respective surgical technique is summarized here briefly. ${ }^{17}$

\section{Conventional TAR $(\mathbf{n}=337)$}

After systemic cooling to a bladder temperature of $25^{\circ} \mathrm{C}$ on cardiopulmonary bypass by the use of unilateral femoral artery perfusion and bicaval drainages, antegrade cerebral perfusion was established by inserting 14-18 Fr balloon catheters into the 3 aortic arch vessels under circulatory arrest. The brachiocephalic artery was clamped to perfuse the right-sided hemisphere in case the right axillary artery also was chosen for arterial cannulation. The aortic arch was transected at the level distal to the aneurysmal end and reconstructed with the use of either the stepwise technique or direct distal anastomosis. ${ }^{5}$ Finally, antegrade systemic perfusion through the rim of the graft was resumed, and 3 cerebral vessels and proximal aorta were reconstructed step-by-step (Video 1).

\section{Debranching With TEVAR $(n=54)$}

For the single debranching technique, a left carotid-left axillary artery bypass with an 8-mm polytetrafluoroethylene (PTFE) graft was performed for zone 2 proximal landing $(\mathrm{n}=23)$, and the double debranching technique, composed of the right axillary-left common carotid and the left axillary bypass with an 8-mm PTFE T-shape graft, was performed for zone 1

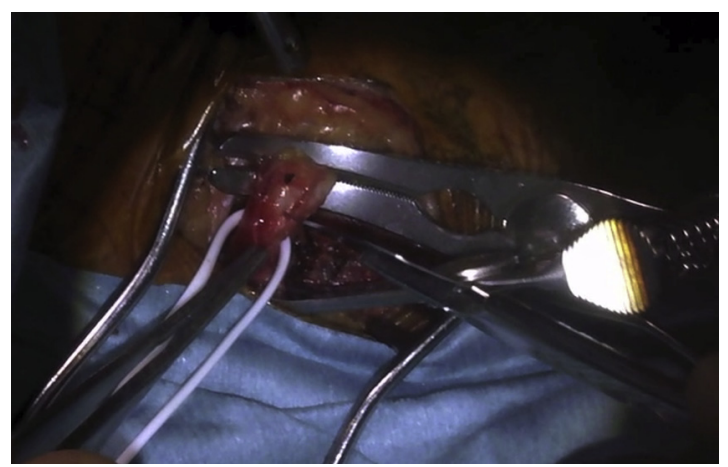

VIDEO 1. Initial-stage arch repair at the proximal site of aneurysm lesion as another option. During systemic cooling under cardiopulmonary bypass using the unilateral femoral (18 Fr) and right axillary arteries (12 Fr), and bicaval cannulations, the neck vessels were taped. Then, Gelweave Siena 4 branch Plexus graft (Vascutek, Inchinnan, Scotland, UK) was trimmed with a 7-cm elephant trunk, and radiopaque marker was placed at the distal edge of the graft. The ascending aorta was clamped, and antegrade cardioplegia was administered. After systemic cooling was achieved to bladder temperature of $25^{\circ} \mathrm{C}$, the brachiocephalic artery was clamped to perfuse the rightsided hemisphere, and antegrade cerebral perfusion was established by inserting 14-18 Fr balloon catheters under circulatory arrest. The aortic arch was transected at the proximal end of the aneurysm and reconstructed using elephant trunk anastomosis placed between the left carotid and left subclavian artery. Then, resuming antegrade systemic perfusion through the rim of the graft, proximal anastomosis was performed and myocardial perfusion resumed. The cerebral vessels were reconstructed step-by-step. Video available at: http://www.jtcvsonline.org/article/S0022-5223(17) 30192-7/addons.

proximal landing $(\mathrm{n}=17)$. The left subclavian artery was embolized routinely for the prevention of persistent type II endoleak after debranching TEVAR. For the total debranching technique, type I hybrid arch repair, which was reported previously by Bavaria and colleagues, ${ }^{10}$ was adopted $(\mathrm{n}=14)$. With regard to device selection, Zenith TX2 (Cook Inc, Bloomington, Ind), Gore TAG or Conformable TAG (W. L. Gore and Associates, Flagstaff, Ariz), and Relay Plus (Bolton Medical Inc, Sunrise, Fla) were used in $12,26,15$, and 1 patients, respectively.

\section{Endovascular Chimney Technique $(n=4)$}

In the patients requiring zone 0 landing, single-stent chimney technique was performed, considering the risk of sternotomy. After a right carotid-left carotid artery bypass with a 7-mm PTFE graft, a chimney graft was deployed in the innominate artery and TEVAR was performed through the femoral artery. The left subclavian artery was embolized routinely for the prevention of persistent type II endoleak and revascularized to the left carotid artery.

\section{Statistical Analysis}

Continuous data are presented as mean \pm standard deviation and were analyzed by the use of 2-tailed $t$-tests or compared with a Mann-Whitney $U$ test for independent data, as appropriate. Categorical variables are given as a count and percentage of patients and compared using the $\chi^{2}$ or Fisher exact test. The survival rate between groups was compared with the Kaplan-Meier model and log-rank test. A $P$ value of $<.05$ was considered significant. All data were analyzed with the Statistical Analysis Systems software JMP 12.0 (SAS Institute Inc, Cary, NC). We also performed propensity-score matching using a $1: 1$ nearest-available matching algorithm with a \pm 0.1 caliper and no 


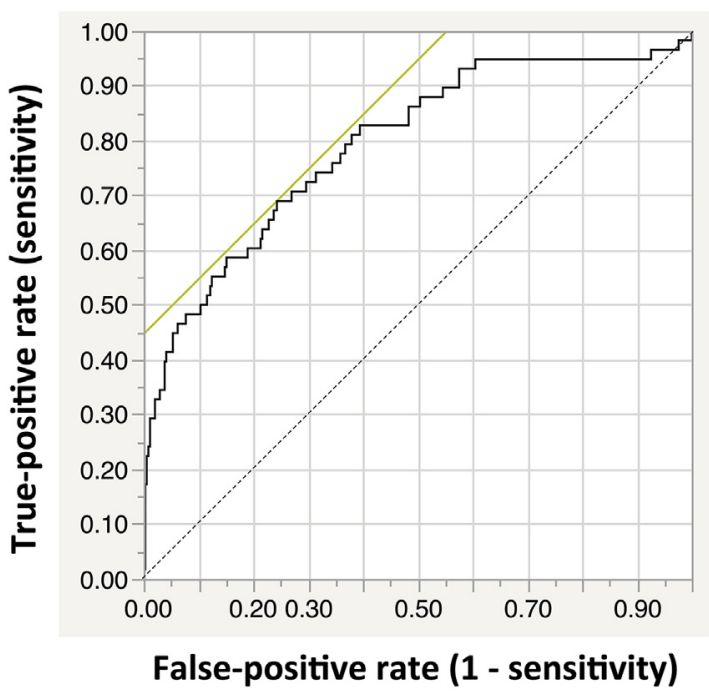

FIGURE 1. The matching model achieved a good discriminatory power $(\mathrm{C}$-statistic $=0.792)$.

replacement for adjustment of significantly different patient characteristics. The analysis yielded 43 (CTAR vs HAR) propensity-score matched observations.

The propensity score was obtained by the use of a multivariate logistic regression model with hybrid approaches as the variables and the 15 baseline characteristics (age, sex, hypertension, dyslipidemia, diabetes mellitus, chronic obstructive pulmonary disease, hemodialysis, peripheral vascular disease, cerebrovascular accident, atherosclerotic change in the aorta, history of previous sternotomy, emergent operation, aortic dissection, ruptured aorta, left ventricular ejection fraction) as covariates based on previous reports. The matching model achieved a good discriminatory power $(\mathrm{C}$-statistic $=0.792$ ) (Figure 1$)$. We compared standardized differences for all covariates between prematch imbalance and postmatch balance. We compared intraoperative and postoperative data using McNemar or conditional logistic regression analysis for categorical variables and paired $t$ test for continuous variables.

\section{RESULTS \\ Unmatched Comparisons of Patient Demographics}

The overall mean patient age was $70.6 \pm 11.5$ (34-92) years, and the percentage of female patients was $30.9 \%$ (122/395). Included were 161 emergency cases (40.8\%). Unmatched comparison between the CTAR and HAR groups revealed a significantly older age of patients $(70.1 \pm 11.6$ years vs $73.6 \pm 11.0$ years; $P=.0294)$ and a significantly greater percentage of patients with chronic obstructive pulmonary disease and history of previous sternotomy $(6.8 \%$ [23/337] vs $15.5 \%$ [9/58]; $P=.0353$ and $1.5 \%$ [5/337] vs $22.4 \%$ [13/58]; $P<.0001$, respectively). In contrast, the percentage of those with emergency operation was significantly greater in the CTAR group compared with the HAR group (43.9\% [148/337] vs $22.4 \%$ [13/58]; $P=.0022)$. Compared with the CTAR group, a significantly lower percentage of aortic dissection and a greater percentage of ruptured aorta in the HAR group was found $(47.5 \%$ [160/337] vs 32.8\% [19/58]; $P=.0453$ and $8.6 \%$ [29/337] vs $19.0 \%$ [11/58]; $P=.0302$, respectively). Unmatched comparisons of preoperative characteristics are shown in Table 1.

\section{Matched Comparisons of Patient Demographics}

By propensity-score analysis, 43 (CTAR vs HAR) matching pairs were obtained (Table 2). Significant imbalance of preoperative characteristics was adjusted well as shown in Figure 2.

\section{Unmatched and Matched Comparisons of Postoperative Data}

The overall 30-day, in-hospital mortality, and operative mortality (30-day, in-hospital and treatment related mortality) were $5.8 \%(23 / 395), 7.6 \%(30 / 395)$, and $8.9 \%(35 /$

TABLE 1. Unmatched comparison of preoperative patient data between CTAR and HAR groups

\begin{tabular}{|c|c|c|c|c|}
\hline & CTAR $(n=337)$ & HAR $(\mathbf{n}=58)$ & $P$ value & Standardized difference \\
\hline Age, y & $70.1 \pm 11.6$ & $73.6 \pm 11.0$ & .0294 & -31.0 \\
\hline Female sex & $105(31.2 \%)$ & $17(29.3 \%)$ & .8781 & 4.1 \\
\hline Hypertension & $276(81.9 \%)$ & $46(79.3 \%)$ & .7140 & 6.6 \\
\hline Dyslipidemia & $99(29.4 \%)$ & $20(34.5 \%)$ & .4413 & -11.0 \\
\hline Diabetes mellitus & $39(11.6 \%)$ & $10(17.2 \%)$ & .2783 & -16.0 \\
\hline COPD & $23(6.8 \%)$ & $9(15.5 \%)$ & .0353 & -27.9 \\
\hline HD patient & $14(4.2 \%)$ & $4(6.9 \%)$ & .3171 & -11.8 \\
\hline PVD & $34(10.1 \%)$ & $6(10.3 \%)$ & 1.0000 & -0.6 \\
\hline CVA & $24(7.1 \%)$ & $8(13.8 \%)$ & .1130 & -22.0 \\
\hline Atherosclerotic aorta & $82(24.3 \%)$ & $18(31.0 \%)$ & .3262 & -15.0 \\
\hline Previous sternotomy & $5(1.5 \%)$ & $58(22.4 \%)$ & $<.0001$ & -68.1 \\
\hline Emergency & $148(43.9 \%)$ & $13(22.4 \%)$ & .0022 & 46.9 \\
\hline Dissection & $160(47.5 \%)$ & $19(32.8 \%)$ & .0453 & 30.3 \\
\hline Ruptured aorta & $29(8.6 \%)$ & $11(19.0 \%)$ & .0302 & -30.5 \\
\hline LVEF, \% & $64.5 \pm 8.8$ & $62.1 \pm 11.1$ & .1166 & 24.0 \\
\hline
\end{tabular}

$C T A R$, Conventional total aortic arch repair; $H A R$, hybrid arch repair; $C O P D$, chronic obstructive pulmonary disease; $H D$, hemodialysis; $P V D$, peripheral vascular disease; $C V A$, cerebrovascular accident; $L V E F$, left ventricular ejection fraction. 
TABLE 2. Matched comparison of preoperative patient data between CTAR and HAR groups

\begin{tabular}{|c|c|c|c|c|}
\hline & CTAR $(n=43)$ & HAR $(n=43)$ & $P$ value & Standardized difference \\
\hline Age, y & $73.9 \pm 8.8$ & $73.2 \pm 10.1$ & .8983 & 7.4 \\
\hline Female sex & $11(25.6 \%)$ & $13(30.2 \%)$ & .4669 & -10.3 \\
\hline Hypertension & $38(88.4 \%)$ & $36(83.7 \%)$ & .7389 & 13.6 \\
\hline Dyslipidemia & $16(37.2 \%)$ & $15(34.9 \%)$ & .8185 & 4.8 \\
\hline Diabetes mellitus & $6(14.0 \%)$ & $7(16.3 \%)$ & .7389 & -6.4 \\
\hline COPD & $6(14.0 \%)$ & $6(14.0 \%)$ & 1.0000 & 0 \\
\hline HD patient & $2(4.7 \%)$ & $2(4.7 \%)$ & 1.0000 & 0 \\
\hline PVD & $6(14.0 \%)$ & $4(9.3 \%)$ & .4795 & 14.7 \\
\hline CVA & $4(9.3 \%)$ & $4(9.3 \%)$ & 1.0000 & 0 \\
\hline Atherosclerotic aorta & $12(27.9 \%)$ & $12(27.9 \%)$ & 1.0000 & 0 \\
\hline Previous sternotomy & $2(4.7 \%)$ & $1(2.3 \%)$ & .3173 & 13.1 \\
\hline Emergency & $13(30.2 \%)$ & $10(23.3 \%)$ & .4054 & 15.6 \\
\hline Dissection & $11(25.6 \%)$ & $14(32.6 \%)$ & .4669 & -15.5 \\
\hline Ruptured aorta & $10(23.3 \%)$ & $8(18.6 \%)$ & .5930 & 11.6 \\
\hline LVEF, \% & $63.4 \pm 7.7$ & $64.7 \pm 7.0$ & .3060 & -17.7 \\
\hline
\end{tabular}

CTAR, Conventional total aortic arch repair; $H A R$, hybrid arch repair; $C O P D$, chronic obstructive pulmonary disease; $H D$, hemodialysis; $P V D$, peripheral vascular disease; $C V A$, cerebrovascular accident; $L V E F$, left ventricular ejection fraction.

395), respectively. Incidences of transient ischemic attack, permanent stroke, and spinal cord injury were $7.1 \%(28 /$ $395), 3.0 \%(12 / 395)$, and $2.8 \%(11 / 395)$, respectively. There was no significant difference in 30-day mortality between the CTAR and HAR groups $(4.8 \%$ [16/337] vs $12.1 \%$ [7/58]; $P=.0602)$; however, in-hospital and operative death was significantly greater in the HAR group compared with the CTAR group (5.6\% [19/337] vs

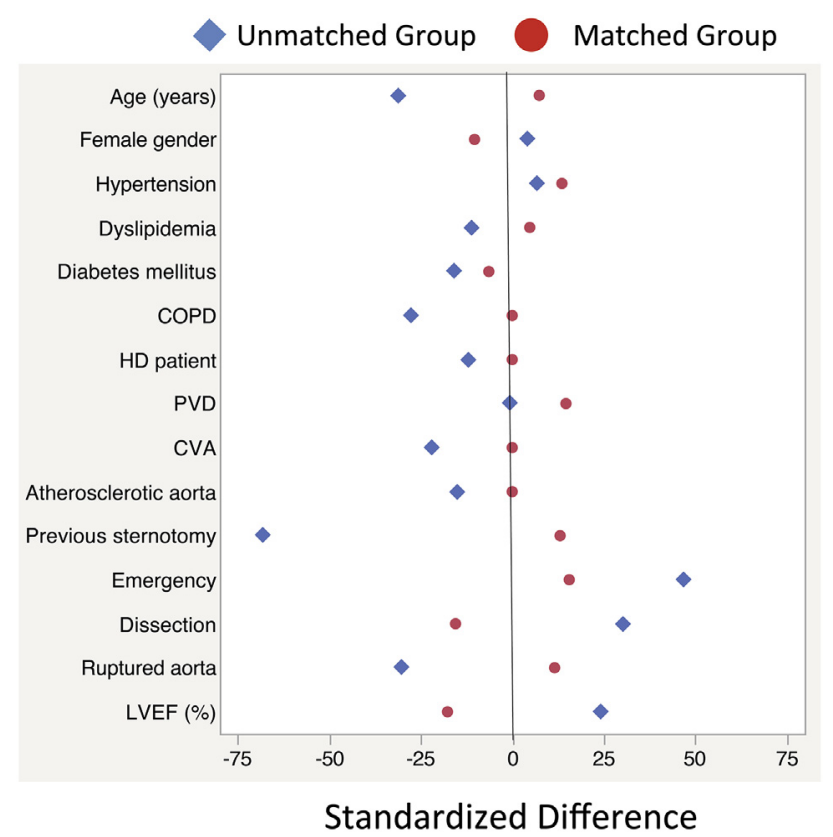

FIGURE 2. Standardized differences. Significant imbalance of preoperative characteristics was adjusted after propensity-score matching. $C O P D$, Chronic obstructive pulmonary disease; $H D$, hemodialysis; $P V D$, peripheral vascular disease; $C V A$, cerebrovascular accident; $L V E F$, left ventricular ejection fraction.
$19.0 \%$ [11/58]; $P=.0016$ and $6.5 \%$ [22/337] vs $22.4 \%$ [13/58]; $P=.0005)$. In addition, unmatched comparison revealed significantly greater incidences of permanent stroke in the HAR group compared with the CTAR group $(1.2 \%$ [4/337] vs $13.8 \%$ [8/58]; $P \leq .0001$ ). Postoperative hospital stay, however, was significantly shorter in the HAR group after we excluded patients with in-hospital death. Unmatched comparisons of postoperative early outcomes are shown in Table E1.

When we used the matched model, significant differences were not shown in 30-day, in-hospital, and operative death between the CTAR and HAR groups $(4.7 \%$ [2/43] vs $7.0 \%$ [3/43], $P=.4142 ; 9.3 \%$ [4/43] vs $14.0 \%$ [6/43], $P=.3173$; and $11.6 \% \quad[5 / 43]$ vs $16.3 \%$ [7/43]; $P=.5637)$. Although there were no significant differences in incidence of transient ischemic attack and spinal cord injury, permanent stroke was observed more frequently in the HAR group compared with the CTAR group $(0 \%[0 /$ 43] vs $11.6 \%$ [5/43]; $P=.0064)$. Matched comparisons of early outcomes between the CTAR and respective HAR groups are shown in Table 3.

\section{Unmatched and Matched Comparisons of Midterm Outcomes}

The average follow-up period was $3.4 \pm 2.6$ years ( $\max$ 10.1 years) in the CTAR group and $1.7 \pm 1.7$ years in the HAR group (max 6.0 years). Follow-up was completed for $98.2 \%$ of patients. In the hybrid groups, reintervention was required in 6 patients. In contrast, there was no patient with reintervention in the CTAR group. Additional TEVAR was performed for a patient with type Ia endoleak. Open repair was required in a patient with new aortic dissection and 3 patients with abnormality of debranched grafts. Coil embolization was performed in a patient with type II 
TABLE 3. Matched comparison of postoperative data between CTAR and HAR groups

\begin{tabular}{lccr}
\hline & CTAR $(\mathbf{n}=\mathbf{4 3})$ & HAR $(\mathbf{n}=\mathbf{4 3})$ & $\boldsymbol{P}$ value \\
\hline 30-d death & $2(4.7 \%)$ & $3(7.0 \%)$ & .4142 \\
In-hospital death & $4(9.3 \%)$ & $6(14.0 \%)$ & .3173 \\
Operative death & $5(11.6 \%)$ & $7(16.3 \%)$ & .5637 \\
Transient ischemic attack & $4(9.3 \%)$ & $5(11.6 \%)$ & .7389 \\
Permanent stroke & $0(0 \%)$ & $5(11.6 \%)$ & .0064 \\
Spinal cord injury & $2(4.7 \%)$ & $2(4.7 \%)$ & 1.0000 \\
Serious infection & $2(4.7 \%)$ & $2(4.7 \%)$ & 1.0000 \\
Hospitalization, d & $29.5 \pm 17.2$ & $27.2 \pm 27.1$ & .8147 \\
\hline
\end{tabular}

CTAR, Conventional total aortic arch repair; $H A R$, hybrid arch repair.

endoleak (Figure E1). Postoperative death during follow-up was seen in 63 patients: 42 patients in the CTAR group and 21 in the HAR group. The causes of death were as follows: 24 aortic events, 8 stroke, 8 respiratory failure, 8 sepsis, 3 cardiac failure, 4 renal failure, 1 liver failure, 2 malignancy, and 5 caducity. In the overall cohort, 5 -year and 10-year survival rate of the CTAR group was $86.7 \%$ and $77.1 \%$, and 5year survival rate of the HAR group was $54.3 \%$. Log-rank test revealed a significantly lower midterm survival rate in the HAR group $(P<.0001)$ (Figure E2). Between the matching pairs, 5-year survival rate of the CTAR and HAR groups was $80.5 \%$ and $59.9 \%$, respectively, and there was no significant difference in the survival rate by log-rank test $(P=.1300)$ (Figure 3, A). In contrast, 5-year and 10year freedom from aorta-related death was $93.8 \%$ and $89.8 \%$, in the CTAR group, and the 5-year freedom from aorta-related death of the HAR group was $91.8 \%$. There was no significant difference by log-rank test $(P=.9262)$ (Figure E3). The matching model also showed no significant differences in freedom from aorta-related death using the log-rank test between the CTAR and HAR group: 5 -year freedom from aorta-related death was $86.9 \%$ in the CTAR group and $90.2 \%$ in the HAR group $(P=.8047)$ (Figure 3, B).

\section{DISCUSSION}

The endovascular technique produced a recognizable change in the strategy of the treatment for aortic pathologies; however, the objective efficiencies of hybrid approaches with TEVAR for aortic arch lesion have not been demonstrated. Therefore, they are still not agreed-on approaches even for high-risk patients. In the present study, matched analysis revealed no significant difference in 30day mortality between the CTAR and HAR groups. Although the matched comparison showed that the incidence rate of permanent stroke was still significantly greater in the HAR group, in-hospital and operative deaths occurred equivalently in the adjusted CTAR and HAR pairs. In addition, matched analysis compensated for the significantly lower midterm survival rate of the HAR group, and the debranching technique had no adverse impacts on freedom from fatal aortic events. However, more meticulous approaches to prevent neurological events should be sought for better outcomes of hybrid approaches with TEVAR.

Several studies reviewed the outcomes of various approaches to aortic arch pathologies, including hybrid repair. Regarding the comparative studies, hybrid TEVAR had been performed in high-risk patients, and efficiencies reported. ${ }^{20-22}$ Benedetto and colleagues, ${ }^{23}$ however,
A

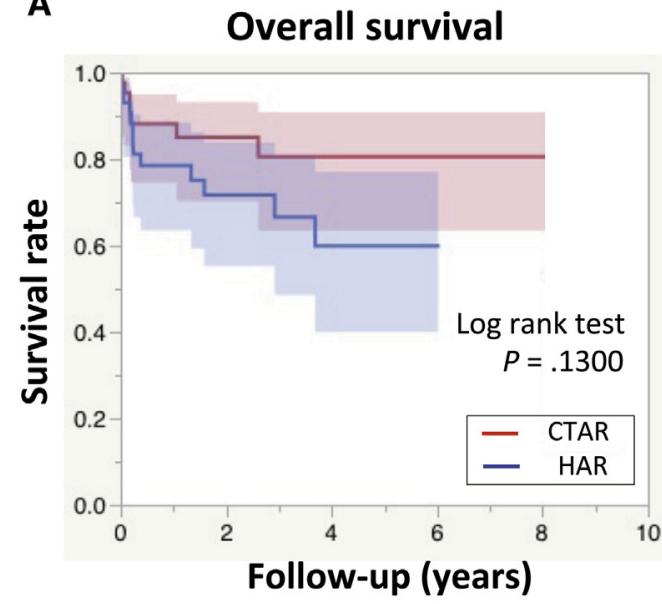

B Freedom from


Number of patients at risk

\begin{tabular}{|lllcl|}
\hline C & 43 & 22 & 15 & 6 \\
H & 43 & 18 & 6 & \\
\hline
\end{tabular}

FIGURE 3. A, Matched comparison of midterm overall survival. Between matching pairs of CTAR and HAR groups, there was no significant difference in the survival rate. B, Matching model also showed no significant differences in freedom from aorta-related death between the CTAR and HAR groups. CTAR, Conventional total aortic arch repair; HAR, hybrid arch repair. 
reported a meta-analysis showing no significant improvement of operative mortality but increased incidence of stroke in patients with hybrid arch repair. Considering the different circumstances of patients, it is difficult to evaluate the real benefits of hybrid technique. Recent publications have been consistently reporting risk-adjusted comparative studies to reduce the influence of different preoperative statuses. Iba and colleagues, ${ }^{18}$ using 35 matched pairs, reported that hybrid arch repair led to significantly shorter intensive care unit and hospital stay but more frequent reintervention. In Tokuda and colleagues, ${ }^{19} 38$ matched pairs yielded no significant differences in early results and significantly lower midterm survival and freedom from aortic event were shown in patients with hybrid approaches. These matched studies did not show better outcomes of hybrid approaches compared with the conventional approach.

By Preventza and colleages, ${ }^{24}$ matched comparison between zone 0 hybrid and open arch repair ( 25 pairs) revealed more neurologic events in the hybrid group but equivalent 4.5 years survival rate. The results are similar to the present study. ${ }^{24}$ Melissano and colleagues ${ }^{25}$ reported that embolism more than hemodynamic hypoperfusion seems to play a major role in the cause of stroke during TEVAR involving aortic arch. The rigid wires and stiff endograft delivery system are thought to be potential for embolization during intervention in diseased aorta. A bare-metal proximal anchoring system is a different risk of stroke after deployment. ${ }^{9,25}$

In our institute, satisfactory outcomes, including permanent stroke incidence and operative mortality in the HAR group, were not achieved between 2006 and 2013, the earlier period $(20.0 \%$ [6/30] and $30.0 \%$ [9/30], respectively). The survival rate of hybrid approaches improved after the introduction of the manual compression of carotid arteries during deployment of the device, the temporary balloon occlusion technique at the time of coil embolization to the left subclavian artery, and careful patient selection as appropriate for hybrid approaches in view of patient frailty and aortic shagginess. Particularly, the indication of proximal landing was determined cautiously, considering the risk of stroke. At the later phase, permanent stroke incidence and operative mortality in the HAR group decreased to $7.1 \%$ [2/28] and $14.3 \%$ [4/28], respectively. Consequently, patient background and learning curve led to improved outcomes of hybrid approaches. Reintervention, however, frequently is required in hybrid TEVAR. Hybrid approaches are developed to be an alternative to conventional open repair but still are not a more advantageous option, especially for high-risk patients.

\section{Study Limitations}

There were several limitations in this study. First, this study was not a prospective randomized analysis.
Therefore, hybrid approaches generally were selected for a certain reason. The selection bias may not be adjusted by propensity-matching, even though the selection of surgical procedure could not be decided randomly and prospectively, when the best approach was sought for each patient. Second, outcomes of an excluded population who had extremely high-risk backgrounds to undergo either conventional and hybrid arch repair were not evaluated. In addition, different indications of hybrid approaches (different ranges and pathologies of aortic lesions) may have influenced on outcomes, although the distal arch lesion could be repaired by both of the CTAR technique and hybrid TEVAR. The range of aortic lesion may be different in some cases between the CTAR and HAR groups; therefore, the difference may influence the outcomes. Lastly, the change of indication of hybrid approaches and learning curve could lead to difficulty in evaluating results.

\section{CONCLUSIONS}

Propensity-score matching analysis revealed significantly greater incidences of postoperative stroke in patients with HAR but equivalent midterm outcomes of hybrid approaches compared with CTAR. In a high-risk population, hybrid approaches have the potential to be alternatives to a conventional approach. Further development, however, will be required for hybrid repair to become a superior option. The optimal strategy, including hybrid approaches, should be sought in accordance with the diverse background of each and every patient.

\section{Conflict of Interest Statement}

Authors have nothing to disclose with regard to commercial support.

\section{References}

1. Okada K, Omura A, Kano H, Sakamoto T, Tanaka A, Inoue T, et al. Recent advancements of total aortic arch replacement. J Thorac Cardiovasc Surg. 2012; 144:139-45.

2. Zierer A, El-Sayed Ahmad A, Papadopoulos N, Moritz A, Diegeler A, Urbanski PP. Selective antegrade cerebral perfusion and mild $\left(28^{\circ} \mathrm{C}-30^{\circ} \mathrm{C}\right)$ systemic hypothermic circulatory arrest for aortic arch replacement: results from 1002 patients. J Thorac Cardiovasc Surg. 2012;144:1042-9.

3. Numata S, Tsutsumi Y, Monta O, Yamazaki S, Seo H, Yoshida S, et al. Mid-longterm results after aortic arch repair using a four-branched graft with antegrade selective cerebral perfusion. J Card Surg. 2013;28:537-42.

4. Urbanski PP, Raad M, Lenos A, Bougioukakis P, Zacher M, Diegeler A. Open aortic arch replacement in the era of endovascular techniques. Eur J Cardiothorac Surg. 2013;44:431-6.

5. Hiraoka A, Chikazawa G, Totsugawa T, Kuinose M, Tamura K, Sakaguchi T, et al. Open total aortic arch reconstruction for patients with advanced age in the era of endovascular repair. J Thorac Cardiovasc Surg. 2014;148:77-82.

6. Urbanski PP, Luehr M, Di Bartolomeo R, Diegeler A, De Paulis R, Esposito G, et al. Multicentre analysis of current strategies and outcomes in open aortic arch surgery: heterogeneity is still an issue. Eur J Cardiothorac Surg. 2016;50: 249-55.

7. Vallejo N, Rodriguez-Lopez JA, Heidari P, Wheatley G, Caparrelli D, Ramaiah V, et al. Hybrid repair of thoracic aortic lesions for zone 0 and 1 in high-risk patients. J Vasc Surg. 2012;55:318-25. 
8. Czerny M, Weigang E, Sodeck G, Schmidli J, Antona C, Gelpi G, et al. Targeting landing zone 0 by total arch rerouting and TEVAR: midterm results of a transcontinental registry. Ann Thorac Surg. 2012;94:84-9.

9. Preventza O, Bakaeen FG, Cervera RD, Coselli JS. Deployment of proximal thoracic endograft in zone 0 of the ascending aorta: treatment options and early outcomes for aortic arch aneurysms in a high-risk population. Eur J Cardiothorac Surg. 2013;44:446-52.

10. Bavaria J, Vallabhajosyula P, Moeller P, Szeto W, Desai N, Pochettino A. Hybrid approaches in the treatment of aortic arch aneurysms: postoperative and midterm outcomes. J Thorac Cardiovasc Surg. 2013;145:S85-90.

11. Shirakawa Y, Kuratani T, Shimamura K, Torikai K, Sakamoto T, Shijo T, et al. The efficacy and short-term results of hybrid thoracic endovascular repair into the ascending aorta for aortic arch pathologies. Eur J Cardiothorac Surg. 2014;45:298-304.

12. Kent WD, Appoo JJ, Bavaria JE, Herget EJ, Moeller P, Pochettino A, et al. Results of type II hybrid arch repair with zone 0 stent graft deployment for complex aortic arch pathology. J Thorac Cardiovasc Surg. 2014;148:2951-5.

13. De Rango P, Cao P, Ferrer C, Simonte G, Coscarella C, Cieri E, et al. Aortic arch debranching and thoracic endovascular repair. J Vasc Surg. 2014;59: 107-14.

14. Faure EM, Canaud L, Marty-Ané C, Alric P. Hybrid aortic arch repair for dissecting aneurysm. J Thorac Cardiovasc Surg. 2016;152:162-8.

15. Vallabhajosyula P, Szeto WY. Current paradigms in aortic arch repair: Striking the balance between open surgery and endovascular repair. J Thorac Cardiovasc Surg. 2015;150:1399-400.

16. Böckler D, Brunkwall J, Taylor PR, Mangialardi N, Hüsing J, Larzon T; CTAG registry investigators. Thoracic endovascular aortic repair of aortic arch pathologies with the conformable thoracic aortic graft: early and 2 year results from a European Multicentre Registry. Eur J Vasc Endovasc Surg. 2016;51:791-800.

17. Hiraoka A, Chikazawa G, Tamura K, Totsugawa T, Sakaguchi T, Yoshitaka H Clinical outcomes of different approaches to aortic arch disease. J Vasc Surg. 2015;61:88-95.
18. Iba Y, Minatoya K, Matsuda H, Sasaki H, Tanaka H, Oda T, et al. How should aortic arch aneurysms be treated in the endovascular aortic repair era? A riskadjusted comparison between open and hybrid arch repair using propensity score-matching analysis. Eur J Cardiothorac Surg. 2014;46:32-9.

19. Tokuda Y, Oshima H, Narita Y, Abe T, Araki Y, Mutsuga M, et al. Hybrid versus open repair of aortic arch aneurysms: comparison of postoperative and mid-term outcomes with a propensity score-matching analysis. Eur J Cardiothorac Surg. 2016;49:149-56.

20. Murashita T, Matsuda H, Domae K, Iba Y, Tanaka H, Sasaki H, et al. Less invasive surgical treatment for aortic arch aneurysms in high-risk patients: a comparative study of hybrid thoracic endovascular aortic repair and conventional total arch replacement. J Thorac Cardiovasc Surg. 2012;143:1007-13.

21. De Rango P, Ferrer C, Coscarella C, Musumeci F, Verzini F, Pogany G, et al. Contemporary comparison of aortic arch repair by endovascular and open surgical reconstructions. J Vasc Surg. 2015;61:339-46.

22. Cao P, De Rango P, Czerny M, Evangelista A, Fattori R, Nienaber C, et al. Systematic review of clinical outcomes in hybrid procedures for aortic arch dissections and other arch diseases. J Thorac Cardiovasc Surg. 2012;144:1286-300.

23. Benedetto U, Melina G, Angeloni E, Codispoti M, Sinatra R. Current results of open total arch replacement versus hybrid thoracic endovascular aortic repair for aortic arch aneurysm: a meta-analysis of comparative studies. J Thorac Cardiovasc Surg. 2013;145:305-6.

24. Preventza O, Garcia A, Cooley DA, Haywood-Watson RJ, Simpson K, Bakaeen FG, et al. Total aortic arch replacement: a comparative study of zone 0 hybrid arch exclusion versus traditional open repair. J Thorac Cardiovasc Surg. 2015;150:1591-8.

25. Melissano G, Tshomba Y, Bertoglio L, Rinaldi E, Chiesa R. Analysis of stroke after TEVAR involving the aortic arch. Eur J Vasc Endovasc Surg. 2012;43: 269-75.

Key Words: aortic arch repair, hybrid arch repair, propensity score matching, thoracic endovascular aortic repair 


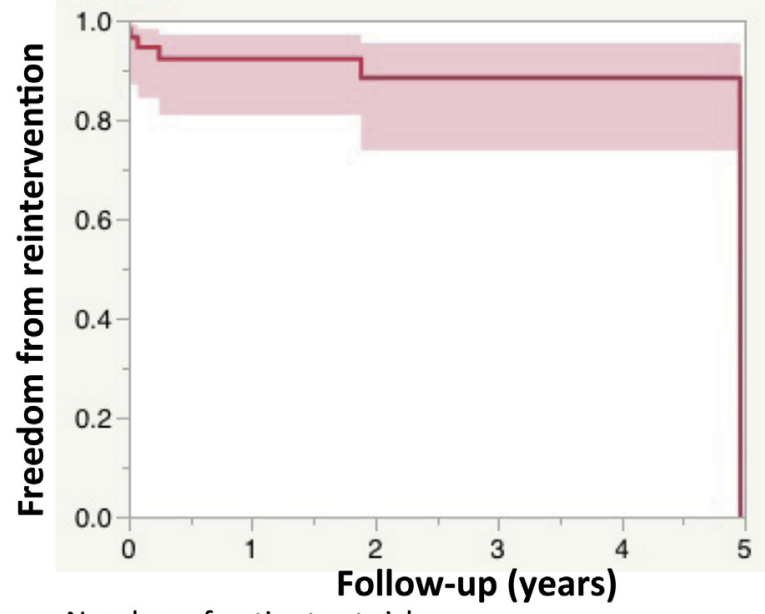

Number of patients at risk

\begin{tabular}{|lllll|}
\hline 58 & 30 & 22 & 15 & 9 \\
\hline
\end{tabular}

FIGURE E1. Freedom from reintervention in patients with hybrid approaches.

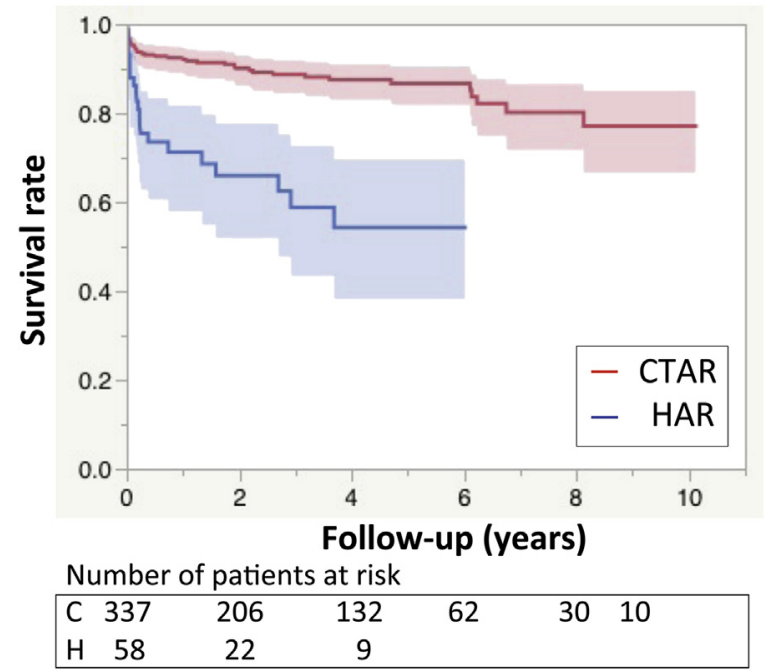

FIGURE E2. Unmatched comparison of midterm survival. Log-rank test revealed a significantly better midterm survival rate in the CTAR group compared with the HAR group $(P<.0001)$. CTAR, Conventional surgical total aortic arch repair; $H A R$, hybrid aortic arch repair.

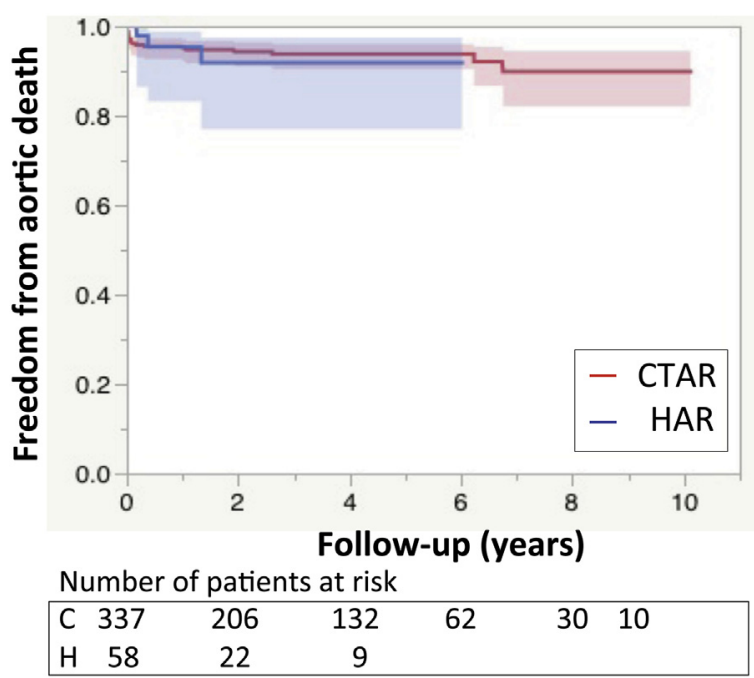

FIGURE E3. Unmatched comparison of freedom from aorta-related death. There was no significant difference between CTAR and the hybrid approach. CTAR, Conventional surgical total aortic arch repair; HAR, hybrid aortic arch repair.

TABLE E1. Unmatched comparison of postoperative data between CTAR and HAR groups

\begin{tabular}{lcc}
\hline & CTAR $(\mathbf{n}=\mathbf{3 3 7})$ & HAR $(\mathbf{n}=\mathbf{5 8})$ \\
\hline 30-d death & $16(4.8 \%)$ & $7(12.1 \%)$ \\
In-hospital death & $19(5.6 \%)$ & $11(19.0 \%)$ \\
Operative death & $22(6.5 \%)$ & $13(22.4 \%)$ \\
Transient ischemic attack & $22(6.5 \%)$ & $6(10.3 \%)$ \\
Permanent stroke & $4(1.2 \%)$ & $8(13.8 \%)$ \\
Spinal cord injury & $9(2.7 \%)$ & $2(3.5 \%)$ \\
Serious infection & $8(2.4 \%)$ & $3(5.2 \%)$ \\
Hospitalization, d & $28.5 \pm 19.3$ & $26.7 \pm 25.0$ \\
\hline CTAR, Conventional surgical total aortic arch repair; HAR, hybrid aortic arch repair.
\end{tabular}

